Supporting Information

\title{
Elastic $\mathrm{Na}_{\mathbf{x}} \mathrm{MoS}_{2}$-carbon-BASE triple interface direct robust solid-solid interface for all-solid-state Na-S batteries
}

Ke Lu, ${ }^{\dagger}$ Bomin Li,${ }^{\dagger}$ Xiaowen Zhan,,${ }^{\dagger}$ Fan Xia,${ }^{\dagger}$ Olusola J. Dahunsi,${ }^{\dagger}$ Siyuan Gao, ${ }^{\dagger}$ David M.

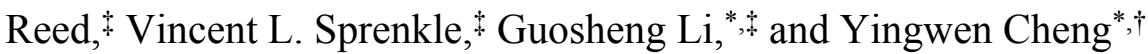

†Department of Chemistry and Biochemistry, Northern Illinois University, DeKalb, IL 60115 Battery Materials and Systems Group, Energy and Environment Directorate, Pacific Northwest National Laboratory, Richland, WA 99354

*E-mails: ycheng@niu.edu (Y.C.); guosheng.li@pnnl.gov (G.L.) 


\section{Materials and Methods}

$\boldsymbol{\beta}$ ''-alumina solid state electrolyte (BASE) fabrication: The composite BASE discs contain $30 \mathrm{vol} \%$ yttria-stabilized zirconia and 70 vol $\% \beta^{\prime \prime}$-alumina, and were fabricated using a tapcasting method followed by a vapor phase conversion and process as has been described in our previous publications. ${ }^{1,2}$ Typical thickness of BASE disc after conversion and creepflattening was $\sim 500 \mu \mathrm{m}$.

Liquid-phase exfoliation of $\mathrm{MoS}_{\mathbf{2}}$ nanosheets: $\mathrm{MoS}_{2}$ nanosheets were obtained by exfoliation of bulk powder ( $<2 \mu \mathrm{m}, 99 \%$, Aldrich) using $\mathrm{NaOH}$ solution in 1-methyl-2pyrrolidinone (NMP, 99\%, Sigma-Aldrich). ${ }^{3}$ Typically, $500 \mathrm{mg} \mathrm{MoS}$, powders were dispersed in $20 \mathrm{ml} \mathrm{N}$-methyl-2-pyrrolidone (NMP) solutions containing $5 \mathrm{mg} \mathrm{NaOH}$. The mixture was sonicated for $4 \mathrm{~h}$ using a Branson bath sonicator and the temperature was controlled between $25^{\circ} \mathrm{C}$ and $30^{\circ} \mathrm{C} . \mathrm{MoS}_{2}$ spontaneously exfoliate via an intercalationexpansion mechanism, and the resulting suspension was collected and was washed with water and ethanol.

Composite anode preparation: The composite Na metal electrodes were prepared in an Arfilled glove box via a thermal melting method. Typically, $667 \mathrm{mg}$ of metallic $\mathrm{Na}$ was loaded in a crucible and was heated to $180^{\circ} \mathrm{C}$ on a hot plate, after the $\mathrm{Na}$ fully melt, $20 \mathrm{mg}$ of dried $\mathrm{MoS}_{2}$ nanoflakes and $20 \mathrm{mg}$ carbon black were added with continuous stirring using a stainless steel rod until homogenous composite was obtained. The mixture was then cooled naturally to room temperature and then solidified ternary composite was obtained, which was pressed into foils using a rolling press for battery assembly and testing.

Sulfur cathode preparation: The sulfur cathodes were prepared by mixing $25 \mathrm{wt} . \%$ $\mathrm{Mo}_{6} \mathrm{~S}_{8} / \mathrm{C}, 65$ wt.\% $\mathrm{S} @ \mathrm{Fe}_{3} \mathrm{O}_{4}-\mathrm{NC}$ powder and 10 wt.\% $\mathrm{PEO}_{10}-\mathrm{NaFSI}$ binder in acetonitrile. $\mathrm{Mo}_{6} \mathrm{~S}_{8}$ nanoparticles, $\mathrm{Fe}_{3} \mathrm{O}_{4}-\mathrm{NC}$ composite and $\mathrm{S} @ \mathrm{Fe}_{3} \mathrm{O}_{4}-\mathrm{NC}$ were synthesized following procedures described in our previous work. ${ }^{4,5}$ The binder was prepared by mixing $40 \mathrm{wt} . \%$ NaFSI and 60 wt.\% PEO (M.W. 5,000,000, Sigma-Aldrich) in acetonitrile and stirring at 60 ${ }^{\circ} \mathrm{C}$ for at least 24 hours. ${ }^{6}$ The PEO powders were vacuum dried at $120{ }^{\circ} \mathrm{C}$ for 5 days prior to use. The slurries were casted on $\mathrm{Al}$ foil and dried at $50{ }^{\circ} \mathrm{C}$ under vacuum for 12 hours, which 
were then punched into $\sim 0.7 \mathrm{~cm}^{2}$ discs and typical sulfur loadings were $\sim 2 \mathrm{mg} \mathrm{cm}^{-2}$. The electrodes were calendared using a rolling press to reduce porosity.

\section{Materials Characterizations}

Powder X-ray diffraction patterns were collected using a Rigaku Miniflex diffractometer $(\mathrm{Cu}$ $\mathrm{K} \alpha \lambda=1.5406 \AA$ ). TEM images were acquired using a field emission JEOL JEM-2100F microscope at $200 \mathrm{KV}$. SEM analysis was performed using a Hitachi S-4700-II microscope equipped with a Bruker EDS detector. Atomic force microscope (AFM) images were using a Bruker MultiMode 8 microscope in tapping mode.

\section{Calculating Young's modulus (E):}

$\mathrm{E}=\sigma / \varepsilon$, where $\sigma=\mathrm{F} / \mathrm{A}, \varepsilon=\Delta \mathrm{L} / \mathrm{L}_{0}$

$\mathrm{F}$ is the force applied to material

$\mathrm{A}$ is the area of cross section

$\mathrm{L}_{0}$ is the original length of the material

$\Delta \mathrm{L}$ is the extended length prior to crack

$\mathrm{E}=\mathrm{F} / \Delta \mathrm{L}$

If we assume $\mathrm{F}$ is similar for both experiments, then Young's modulus for $\mathrm{Na}$ and $\mathrm{Na}-\mathrm{C}$ $\mathrm{Na}_{\mathrm{x}} \mathrm{MoS}_{2}$ depends on $\Delta \mathrm{Ls}$. From our experiments, the measured $\Delta \mathrm{Ls}$ are 0.6 and $1.4 \mathrm{~cm}$ for pristine $\mathrm{Na}$ and $\mathrm{Na}-\mathrm{C}-\mathrm{Na}_{\mathrm{x}} \mathrm{MoS}_{2}$, respectively. Therefore the Young's modulus of Na-C$\mathrm{Na}_{\mathrm{x}} \mathrm{MoS}_{2}$ is approximately 2 times smaller than that of pristine Na. Smaller Young's modulus generally indicates less stiffness with higher elasticity.

\section{Electrochemical studies}

The batteries were assembled in an Ar-filled MBraun LabStar glovebox where oxygen and moisture levels were both below $0.5 \mathrm{ppm}$. The batteries were cycled with a voltage range of $0.5 \sim 2.5 \mathrm{~V}$ using a Neware BTS-4000 battery analyzer at desired current densities.

Electrochemical impedance spectra were acquired using a $\mathrm{CH}$ Instruments potentiostat using the frequency range of $100 \mathrm{kHz}$ to $100 \mathrm{mHz}$. A Tenney TJR environmental chamber was used to control temperatures for all battery cycling and electrochemical analysis. 


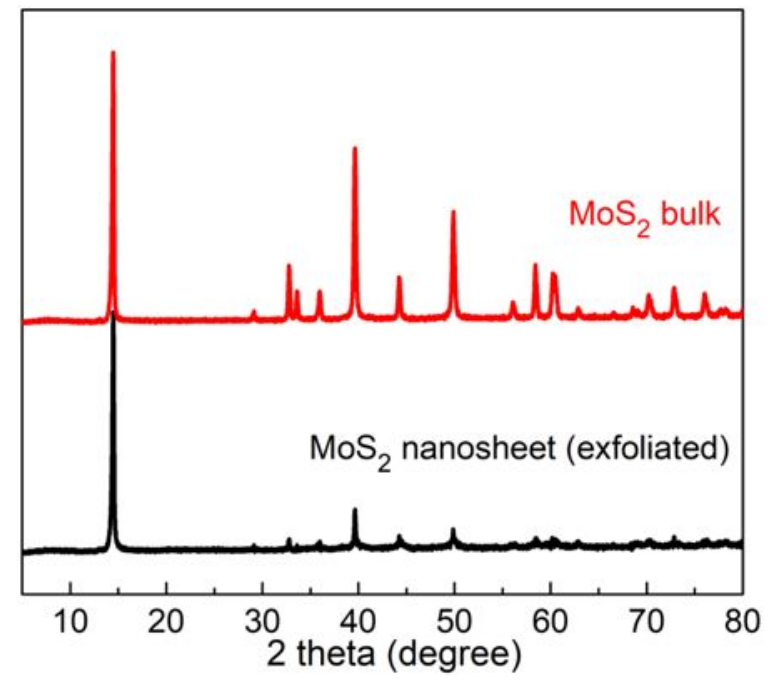

Figure S1: Comparison of X-ray diffraction patterns of pristine $\mathrm{MoS}_{2}$ powder and exfoliated $\mathrm{MoS}_{2}$ nanosheets.

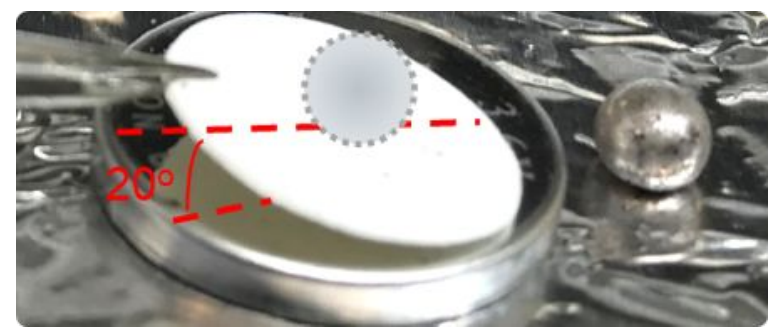

Figure S2: Photograph image that shows the highly sodiophobic properties of BASE with pristine molten $\mathrm{Na}$. 

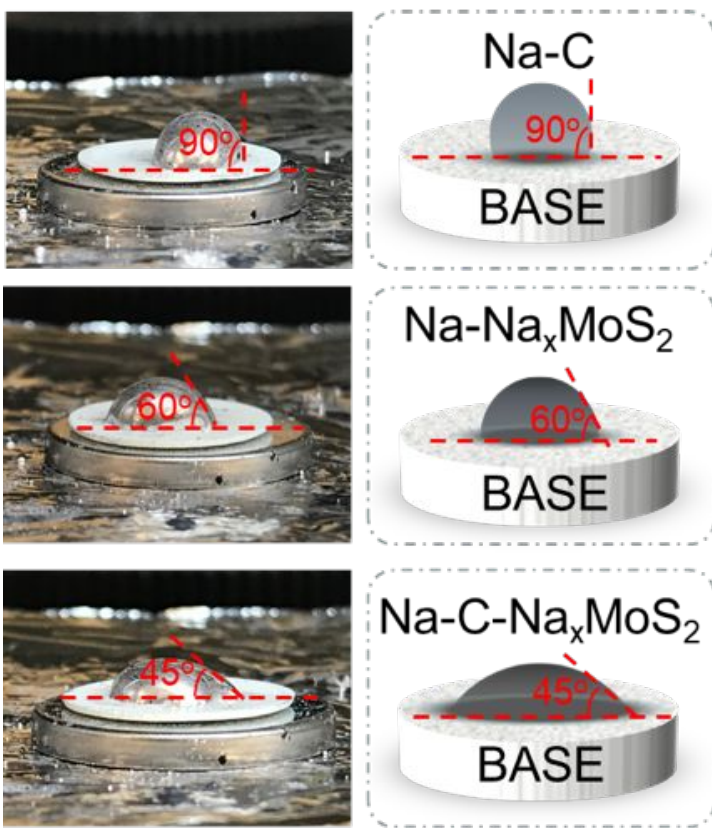

Figure S3: Comparison of contact angles of different Na composite on BASE.



Figure S4: Nyquist plot of solid-state $\mathrm{Na} / \mathrm{Na}$ symmetric cells at various temperatures. 


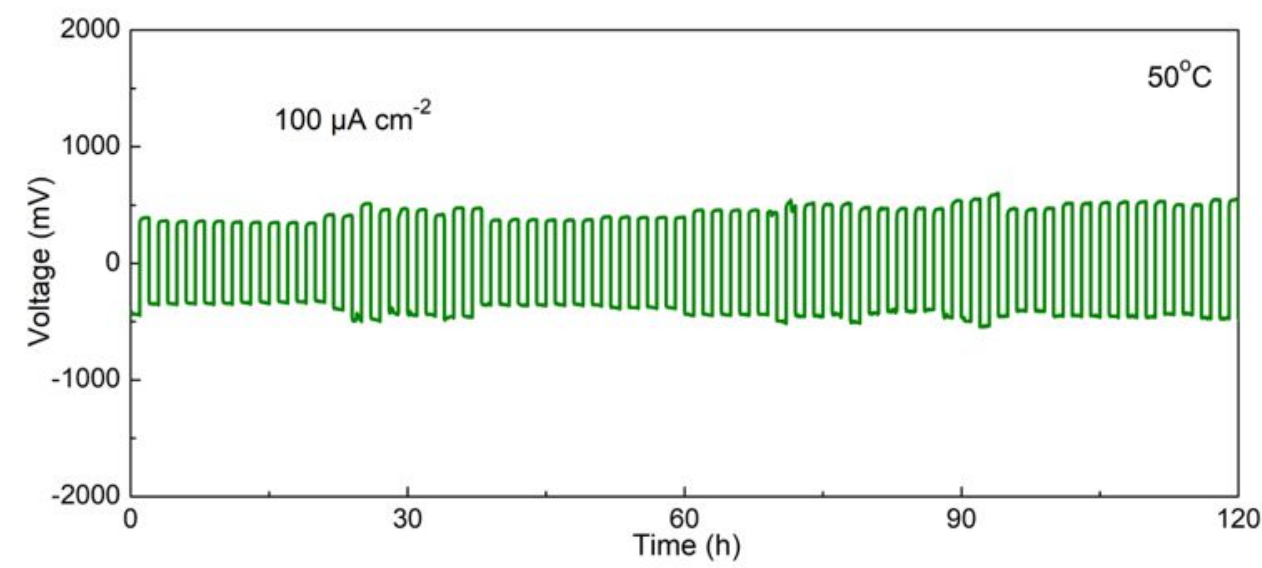

Figure S5: Long-term cycling stability of symmetric $\mathrm{Na}^{+} / \mathrm{Na}$ via solid-state triple junction interface on BASE at $50^{\circ} \mathrm{C}$.

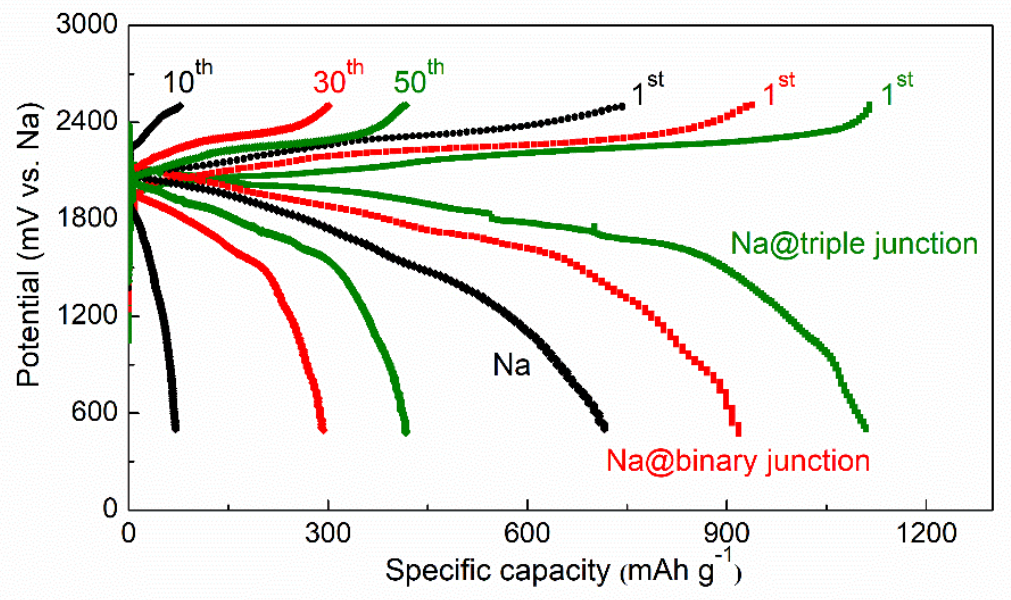

Figure S6: Comparison of voltage profiles of full cells with different anodes at relevant cycles. The batteries were cycled at $0.2 \mathrm{~mA} \mathrm{~cm}^{-2}$ and $80^{\circ} \mathrm{C}$. 


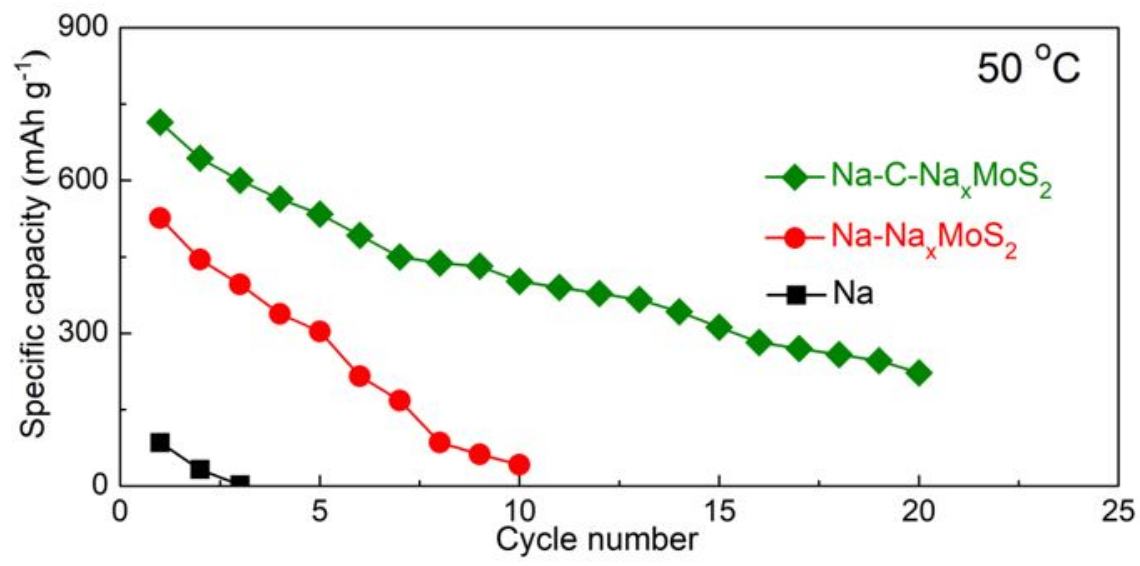

Figure S7: Cycling stability of ASS Na-S cells equipped with different Na anode at $50^{\circ} \mathrm{C}$.

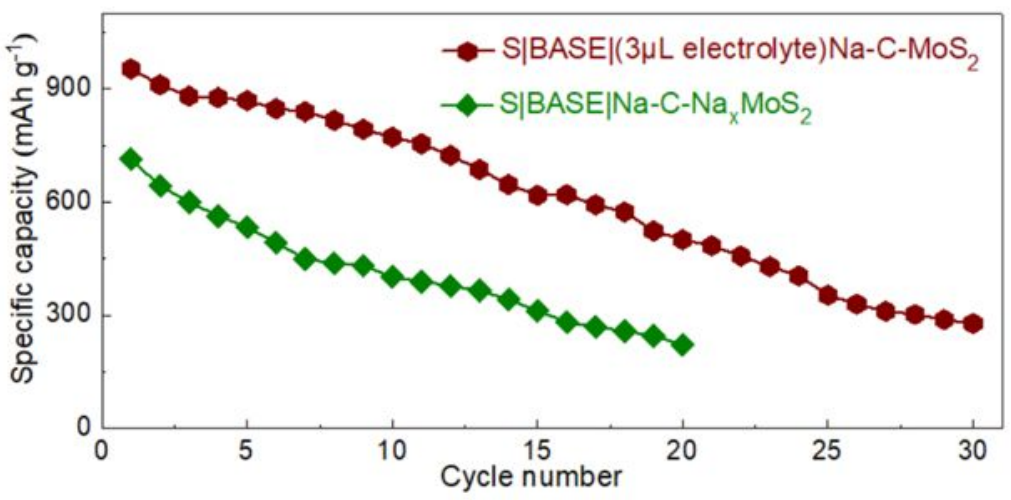

Figure S8: Long-term stability of cycling Na-S solid-state cells with and without adding liquid electrolyte at $50^{\circ} \mathrm{C}$.

Table S1. Comparison recent advances of BASE-base All-Solid-State Na metal batteries

\begin{tabular}{cccc}
\hline Cell & $\begin{array}{c}\text { Working potential } \\
(\mathbf{V} \text { vs. Na) }\end{array}$ & Working temperature & Citation \\
\hline $\mathbf{N a} / \mathbf{B A S E} / \mathbf{S}$ & $\mathbf{0 . 5}-\mathbf{2 . 5}$ & $\mathbf{8 0}^{\circ} \mathbf{C}$ & Our work \\
$\mathrm{Na} / \mathrm{BASE} / \mathrm{NiCl}_{2}$ & $2.4-2.8$ & $175-300^{\circ} \mathrm{C}$ & 7 \\
$\mathrm{Na} / \mathrm{BASE} / \mathrm{NiCl}_{2}$ & $1.7-2.4$ & $190^{\circ} \mathrm{C}$ & 8 \\
$\mathrm{Na} / \mathrm{BASE} / \mathrm{FeCl}_{2}$ & $1.8-2.6$ & $190^{\circ} \mathrm{C}$ & 2 \\
\hline
\end{tabular}




\begin{tabular}{llll}
\hline $\mathrm{Na} / \mathrm{BASE} / \mathrm{NiCl}_{2}$ & $1.8-2.8$ & $175^{\circ} \mathrm{C}$ & 9 \\
\hline
\end{tabular}

Table S2. Comparison recent advances of solid state Na-S batteries

\begin{tabular}{|c|c|c|c|c|c|}
\hline Electrolyte & $\begin{array}{c}\text { Average } \\
\text { discharge voltage } \\
\text { (V vs. Na) }\end{array}$ & Temperature & $\begin{array}{l}\text { Capacity } \\
(\mathbf{m A h} / \mathrm{g})\end{array}$ & $\begin{array}{c}\text { Capacity } \\
\text { retention/cycles }\end{array}$ & Citation \\
\hline BASE-No liquid & 1.8 & $8^{\circ} \mathrm{C}$ & 1110 & $37.4 \% / 50$ & $\begin{array}{c}\text { Our } \\
\text { work }\end{array}$ \\
\hline $\mathrm{Na}_{3} \mathrm{SbS}_{4}$-No liquid & 0.5 & r.t. & 1560 & $93 \% / 50$ & 10 \\
\hline $\begin{array}{c}\mathrm{Na}_{3} \mathrm{Zr}_{2} \mathrm{Si}_{2} \mathrm{PO}_{12}-5 \mu \mathrm{l} \\
\text { liquid }\end{array}$ & 1.8 & r.t. & 1000 & $78.6 \% / 100$ & 11 \\
\hline $\begin{array}{c}\mathrm{PEO}-\mathrm{NaCF}_{3} \mathrm{SO}_{3}- \\
\text { MIL-53(Al)-No } \\
\text { liquid }\end{array}$ & 1.5 & $60^{\circ} \mathrm{C}$ & 897.7 & $75.2 \% / 50$ & 12 \\
\hline $\mathrm{Na}_{3} \mathrm{PS}_{4}$-No liquid & 1.2 & $60^{\circ} \mathrm{C}$ & 850 & $76.5 \% / 50$ & 13 \\
\hline $\mathrm{Na}_{3} \mathrm{PS}_{4}$-No liquid & 1.2 & r.t. & 1500 & $50 \% / 2$ & 14 \\
\hline $\mathrm{Na}_{3} \mathrm{PS}_{4}$-No liquid & 1.5 & $60^{\circ} \mathrm{C}$ & 869.2 & $50.4 \% / 50$ & 15 \\
\hline $\mathrm{Na}_{3} \mathrm{SbS}_{4}-\mathrm{No}$ liquid & 1.0 & r.t. & 316 & $88.2 \% / 150$ & 16 \\
\hline PFSA-Na-No liquid & 1.1 & r.t. & 461.2 & $62 \% / 50$ & 17 \\
\hline $\mathrm{Na}_{3} \mathrm{SbS}_{4}$-No liquid & 1.4 & r.t. & 1386.3 & $56.7 \% / 100$ & 18 \\
\hline $\begin{array}{c}\mathrm{Na}_{3} \mathrm{PS}_{4}-\mathrm{Pyr}_{14} \mathrm{FSI}(\mathrm{IL}, \\
\mathrm{SEI})\end{array}$ & 1.4 & r.t. & 717.3 & $80.8 \% / 50$ & 19 \\
\hline $\begin{array}{l}\text { Gel polymer-No } \\
\text { liquid }\end{array}$ & 1.3 & r.t. & 877 & $83.9 \% / 100$ & 20 \\
\hline PVdF-No liquid & 1.8 & r.t. & 392 & $29.8 \% / 10$ & 21 \\
\hline $\begin{array}{c}\text { PVdF-HCF gel-No } \\
\text { liquid }\end{array}$ & 1.4 & r.t. & 165 & $12.7 \% / 8$ & 22 \\
\hline PEO gel-No liquid & 1.8 & $90^{\circ} \mathrm{C}$ & 500 & $33 \% / 10$ & 23 \\
\hline
\end{tabular}




\section{References}

1. Jung, K.; Chang, H.-J.; Bonnett, J. F.; Canfield, N. L.; Sprenkle, V. L.; Li, G., An advanced $\mathrm{Na}-\mathrm{NiCl}_{2}$ battery using bi-layer (dense/micro-porous) $\beta$ "-alumina solid-state electrolytes. Journal of Power Sources 2018, 396, 297-303.

2. Zhan, X.; Bowden, M. E.; Lu, X.; Bonnett, J. F.; Lemmon, T.; Reed, D. M.; Sprenkle, V. L.; Li, G., A Low-Cost Durable Na-FeCl 2 Battery with Ultrahigh Rate Capability. Advanced Energy Materials 2020, 10 (10), 1903472.

3. Bang, G. S.; Nam, K. W.; Kim, J. Y.; Shin, J.; Choi, J. W.; Choi, S.-Y., Effective LiquidPhase Exfoliation and Sodium Ion Battery Application of $\mathrm{MoS}_{2}$ Nanosheets. ACS Applied Materials \& Interfaces 2014, 6 (10), 7084-7089.

4. Lu, K.; Liu, Y.; Chen, J.; Zhang, Z.; Cheng, Y., Redox Catalytic and Quasi-Solid Sulfur Conversion for High-Capacity Lean Lithium Sulfur Batteries. ACS Nano 2019, 13 (12), 14540-14548.

5. Lu, K.; Zhang, H.; Gao, S.; Ma, H.; Chen, J.; Cheng, Y., Manipulating Polysulfide Conversion with Strongly Coupled $\mathrm{Fe}_{3} \mathrm{O}_{4}$ and Nitrogen Doped Carbon for Stable and High Capacity Lithium-Sulfur Batteries. Advanced Functional Materials 2019, 29 (4), 1807309.

6. Chen, J.; Henderson, W. A.; Pan, H.; Perdue, B. R.; Cao, R.; Hu, J. Z.; Wan, C.; Han, K. S.; Mueller, K. T.; Zhang, J.-G.; Shao, Y.; Liu, J., Improving Lithium-Sulfur Battery Performance under Lean Electrolyte through Nanoscale Confinement in Soft Swellable Gels. Nano Letters 2017, 17 (5), 3061-3067.

7. Jin, D.; Choi, S.; Jang, W.; Soon, A.; Kim, J.; Moon, H.; Lee, W.; Lee, Y.; Son, S.; Park, Y.-C.; Chang, H.; Li, G.; Jung, K.; Shim, W., Bismuth Islands for Low-Temperature SodiumBeta Alumina Batteries. ACS Applied Materials \& Interfaces 2019, 11 (3), 2917-2924.

8. Lu, X.; Chang, H. J.; Bonnett, J. F.; Canfield, N. L.; Jung, K.; Sprenkle, V. L.; Li, G., An Intermediate-Temperature High-Performance $\mathrm{Na}-\mathrm{ZnCl}_{2}$ Battery. ACS Omega 2018, 3 (11), 15702-15708.

9. Jin, D.; Lee, H. G.; Choi, S.; Kim, S.; Lee, Y.; Son, S.; Park, Y.-C.; Lee, J. S.; Jung, K.; Shim, W., Sparked Reduced Graphene Oxide for Low-Temperature Sodium-Beta Alumina Batteries. Nano Letters 2019, 19 (12), 8811-8820.

10. Ando, T.; Sakuda, A.; Tatsumisago, M.; Hayashi, A., All-solid-state sodium-sulfur battery showing full capacity with activated carbon $\mathrm{MSP}_{20}$-sulfur- $\mathrm{Na}_{3} \mathrm{SbS}_{4}$ composite. Electrochemistry Communications 2020, 116, 106741.

11. Yu, X.; Manthiram, A., Sodium-Sulfur Batteries with a Polymer-Coated NASICON-type Sodium-Ion Solid Electrolyte. Matter 2019, 1 (2), 439-451.

12. Ge, Z.; Li, J.; Liu, J., Enhanced electrochemical performance of all-solid-state sodiumsulfur batteries by PEO-NaCF $3 \mathrm{SO}_{3}-\mathrm{MIL}-53(\mathrm{Al})$ solid electrolyte. Ionics 2020, 26 (4), 1787 1795.

13. Fan, X.; Yue, J.; Han, F.; Chen, J.; Deng, T.; Zhou, X.; Hou, S.; Wang, C., High-

Performance All-Solid-State Na-S Battery Enabled by Casting-Annealing Technology. ACS Nano 2018, 12 (4), 3360-3368. 
14. Nagata, H.; Chikusa, Y., An All-solid-state Sodium-Sulfur Battery Operating at Room Temperature Using a High-sulfur-content Positive Composite Electrode. Chemistry Letters 2014, 43 (8), 1333-1334.

15. Yue, J.; Han, F.; Fan, X.; Zhu, X.; Ma, Z.; Yang, J.; Wang, C., High-Performance AllInorganic Solid-State Sodium-Sulfur Battery. ACS Nano 2017, 11 (5), 4885-4891.

16. Zhang, Z.; Cao, H.; Yang, M.; Yan, X.; Yu, C.; Liu, D.; Zhang, L., High performance room temperature all-solid-state $\mathrm{Na}-\mathrm{Se}_{\mathrm{x}} \mathrm{S}$ battery with $\mathrm{Na}_{3} \mathrm{SbS}_{4}$-coated cathode via aqueous solution. Journal of Energy Chemistry 2020, 48, 250-258.

17. Ma, Q.; Du, G.; Guo, B.; Tang, W.; Li, Y.; Xu, M.; Li, C., Carbon-wrapped cobalt nanoparticles on graphene aerogel for solid-state room-temperature sodium-sulfur batteries. Chemical Engineering Journal 2020, 388, 124210.

18. Wan, H.; Weng, W.; Han, F.; Cai, L.; Wang, C.; Yao, X., Bio-inspired Nanoscaled Electronic/Ionic Conduction Networks for Room-Temperature All-Solid-State Sodium-Sulfur Battery. Nano Today 2020, 33, 100860.

19. An, T.; Jia, H.; Peng, L.; Xie, J., Material and Interfacial Modification toward a Stable Room-Temperature Solid-State Na-S Battery. ACS Applied Materials \& Interfaces 2020, 12 (18), 20563-20569.

20. Zhou, D.; Chen, Y.; Li, B.; Fan, H.; Cheng, F.; Shanmukaraj, D.; Rojo, T.; Armand, M.; Wang, G., A Stable Quasi-Solid-State Sodium-Sulfur Battery. Angewandte Chemie International Edition 2018, 57 (32), 10168-10172.

21. Kim, J.-S.; Ahn, H.-J.; Kim, I.-P.; Kim, K.-W.; Ahn, J.-H.; Park, C.-W.; Ryu, H.-S., The short-term cycling properties of $\mathrm{Na} / \mathrm{PVdF} / \mathrm{S}$ battery at ambient temperature. Journal of Solid State Electrochemistry 2008, 12 (7), 861-865.

22. Kumar, D.; Suleman, M.; Hashmi, S. A., Studies on poly(vinylidene fluoride-cohexafluoropropylene) based gel electrolyte nanocomposite for sodium-sulfur batteries. Solid State Ionics 2011, 202 (1), 45-53.

23. Park, C.-W.; Ryu, H.-S.; Kim, K.-W.; Ahn, J.-H.; Lee, J.-Y.; Ahn, H.-J., Discharge properties of all-solid sodium-sulfur battery using poly (ethylene oxide) electrolyte. Journal of Power Sources 2007, 165 (1), 450-454. 